\title{
Effect of Semi-Recumbent Position on Blood Oxygen Saturation in Postanesthetic Patients After Laparoscopic Cholecystectomy
}

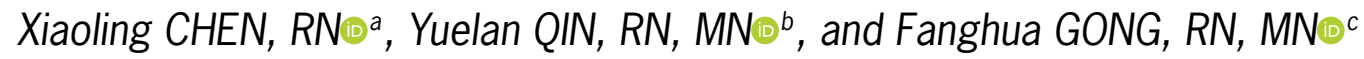

Background:Undergoing general anesthesia and pneumoperitoneum could affect the lung function and comprise oxygenation in laparoscopic cholecystectomy patients. Position of patients after surgery may affect lung function of these patients. Aim: To observe the effect of semi-recumbent position on blood oxygen saturation $\left(\mathrm{SpO}_{2}\right)$ in postanesthetic patients after laparoscopic cholecystectomy. Methods: $A$ number of 225 patients who admitted to postanesthetic care unit after laparoscopic cholecystectomy from September 2016 to May 2017 were randomly assigned into the intervention group and the control group. The intervention group adopted a semi-recumbent position with head bed elevation by 10 to $20^{\circ}$, while the control received a supine position without lying on a pillow. Both groups received routine postanesthesia care. Data on $\mathrm{SpO}_{2}$ before and after extubation were collected. Independent $t$-test was used for data analysis. Results: There was no vomiting and cough reactions before and after extubation in both groups. Intervention group reported a significantly higher level of $\mathrm{SpO}_{2}$ at 1 minute and 30 minutes after extubation, compared to the control group (both ps <0.05). Conclusion: Semi-recumbent position with the head bed elevation could significantly increase the $\mathrm{SpO}_{2}$ level of postanesthetic patients, thus ensuring the safety of patients when removing the endotracheal tube.

Keywords: position; postanesthetic care; laparoscopic cholecystectomy; blood oxygen saturation

\section{BACKGROUND}

Laparoscopic cholecystectomy involves the use of a laparoscope with instrument insertion. This procedure has been widely used as a routine procedure for gallbladder removal (Wolfe, Gardiner, \& Frey, 2015). It has become one of the most commonly performed general surgical procedure in the United States (Wolfe et al., 2015). Various advantages of laparoscopic approach contribute to its popularity, including the decreased postoperative pain, postoperative morbidity, and mortality, shorten hospital stay, rapid recovery, and being superior in cosmetic results when compared with the traditional or open cholecystectomy (Coccolini et al., 2015; Nasser et al., 2015). A standard laparoscopic cholecystectomy requires a condition of being general anesthesia of patients (Nathaniel \& Preeti, 2018), consequently, hypoxemia is likely to occur due to the consequences of anesthesia and surgery impacting on lung function (Saraswat, 2015). The position of a patient may affect respiratory function and thus influence oxygen saturation (Saraswat, 2015). Blood oxygen saturation is an important

\footnotetext{
${ }^{a}$ The First People's Hospital of Changde City, Changde, Hunan, China, Email: 2292572987@qq.com

${ }^{b}$ People's Hospital of Hunan Province, Changsha, Hunan, China

${ }^{c}$ People's Hospital of Hunan Province, Changsha, Hunan, China
} 
physiological parameter of the respiratory cycle, and monitoring blood oxygen saturation could facilitate the detection of hypoxia which also provides a reliable basis for the change of lung condition and allow the initiation of timely treatment (Chen, 2015; Jiang, Zhao, \& Liu, 2013; Zhang, 2014). However, the conclusion on an optimal position to improve blood oxygen saturation is yet to be achieved (Saraswat, 2015). Therefore, this study aimed to examine the effect of patient position on the blood oxygen saturation of postanesthetic patients after laparoscopic cholecystectomy.

\section{METHODS}

\section{Participants}

Patients received laparoscopic cholecystectomy under general anesthesia from September 2016 to May 2017 in our hospital were randomly assigned to the control group or the intervention group according to the time sequence of admission to the postanesthetic care unit (PACU) which is a place for intensive observation and monitoring of patients after anesthesia for a short period of time. Those who admitted to PACU on an even date were randomized to intervention group, while control group comprised those who admitted to PACU on an odd date. Patients who met the following criteria were invited to participate in this study. Inclusion criteria: 1) undergoing general anesthesia; 2) normal preoperative cardiac and pulmonary function; and 3) orotracheal intubation. Patients were excluded if they were having 1 ) upper respiratory tract infection; 2) cognitive impairment; and 3) history of mental illness.

\section{Procedure}

After entering the PACU, eligible patients were invited to participate in this study and informed consent was obtained from the patient's family. All patients received ventilator-assisted breathing (SIMV mode, oxygen concentration 40\%) and usual care. Patients in the control group were placed in a supine position without lying on a pillow. While patients in the intervention group were in a semi-recumbent position by raising the head bed by 10 to $20^{\circ}$. The endotracheal tube was removed by the doctor when patients recovered from unconsciousness with stable vital signs and meeting the criteria for extubation. After removing the endotracheal tube, all patients received oxygen through the nasal cannula and closely monitoring of vital signs.

\section{Measurements}

Pulse oximetry $\left(\mathrm{SpO}_{2}\right)$ was used to evaluate the oxygen status of patients by putting an oximeter finger probe on a thumb of the patient. As the oximeter needs a flow of blood through the finger to function, the affected limb was not used for measuring blood pressure. The values of $\mathrm{SpO}_{2}$ were recorded at $1 \mathrm{~min}$ before, and $1 \mathrm{~min}$ and 30 min after extubation. Other complications associated with extubation such as cough and vomiting were also recorded.

\section{Data Analysis}

SPSS 21.0 software was used for statistical analysis. Descriptive statistics such as percentage, mean (standard deviation) were used to present the data. Chi-square test, independent $t$-test, or Kruskal Wallis test was used to examine the differences between two groups. $p<0.05$ was considered statistically significant.

\section{RESULTS}

A total of 225 patients were recruited, with 110 in the control group and 115 in the intervention group. There was no statistical difference in age, gender, body mass index (BMI), duration of operation, American Society of Anesthesiologists (ASA) physical status classification (all $p>0.05$ ) (Table 1).

No sedative analgesic was used in both groups during the period in PACU. In both groups, $\mathrm{SpO}_{2}$ was $100 \%$ at $1 \mathrm{~min}$ before extubation. After extubation, $\mathrm{SpO}_{2}$ at 1 minute and 30 minutes were significantly higher in the intervention group compared with the control group (all $p<0.05$ ) (Table 2). No vomiting nor coughing at before or after extubation was observed in both groups. 
TABLE 1. Sociodemographic Characteristics of Participants

\begin{tabular}{lllll}
\hline Variables & & Control Group (n= 110) & Intervention Group (n = 1 15) & $\boldsymbol{p}$ \\
\hline Age (years) & & $51.20 \pm 12.39$ & $51.36 \pm 10.46$ & 0.918 \\
Sex & male & 64 & 68 & 0.885 \\
& female & 46 & 47 & 0.456 \\
BMI & & $23.16 \pm 4.18$ & $23.57 \pm 4.00$ & 0.125 \\
Duration of & $83.05 \pm 36.28$ & $91.30 \pm 43.48$ & \\
operation & & & 0.067 \\
(minutes) & & 95 & 100 & 7 \\
ASA classification & I & II & 13 & 8 \\
& & III & 2 & 8 \\
\hline
\end{tabular}

Note. ASA I = a normal healthy patient; ASA II = a patient with mild systemic disease; ASA III = a patient with severer systemic disease; BMI = body mass index.

TABLE 2. Comparison on $\mathrm{SpO}_{2}$ Before and After Extubation Between Groups

\begin{tabular}{lllll}
\hline Time & Control Group $(\boldsymbol{n}=\mathbf{1 1 0})$ & Intervention Group $(\boldsymbol{n}=\mathbf{1 1 5})$ & $\boldsymbol{t}$ & $\boldsymbol{p}$ \\
\hline $\begin{array}{l}1 \text { min before } \\
\text { extubation }\end{array}$ & 100 & 100 & - & - \\
$\begin{array}{l}1 \text { min after } \\
\text { extubation }\end{array}$ & $92.84 \pm 1.88$ & $98.57 \pm 1.70$ & $23.999<0.001$ \\
\begin{tabular}{l}
$\begin{array}{l}\text { 30 min after } \\
\text { extubation }\end{array}$ \\
\hline
\end{tabular} & $97.84 \pm 2.35$ & $99.87 \pm 0.13$ & 9.250 & $<0.001$ \\
\hline
\end{tabular}

\section{DISCUSSION}

This study examined the effect of different positions on $\mathrm{SpO}_{2}$ level in postanesthetic patients after laparoscopic cholecystectomy. The results showed that the semi-recumbent position with head bed elevated by 10 to $20^{\circ}$ could significantly increase $\mathrm{SpO}_{2}$ level after extubation than the supine position.

General anesthesia could impair gases exchange and affect respiratory mechanics of a patient (Yousef \& Lasheen, 2013). Additionally, pneumoperitoneum, a required procedure for laparoscopic cholecystectomy, may cause respiratory changes such as increased arterial pressure of $\mathrm{CO}_{2}$ and a negative impacting vital capacity (Longo, Cavalheiro, \& de Oliveira Filho, 2017), which could further limit the lung function.

The diaphragm is the most important respiratory muscle in the body's respiratory movement.
The tidal volume produced during the quiet status accounts for about $80 \%$ of the total tidal volume (Gao, Li, \& Cheng, 2010). Studies have found that in postoperative patients undergoing general anesthesia, a semi-recumbent position could increase their tidal volume than the supine position due to the lowering of diaphragm and the expanding of alveolar, thus increasing oxygenation (Pinto, Eriyawa, Weerasinghe, Senasinghe, \& Rajendran, 2013), which are consistent with the findings of the current study.

However, several limitations of this study may affect the generalization of the results. The uniqueness of the study unit using supine position without pillow may not be a practice in many other PACU. In addition, the randomization of this study using odd/even day approach may not be the true randomization method. As such, confounding variables in patients between the two groups may also affect the trustworthiness of the results. 


\section{CONCLUSION}

The position can affect the respiratory function of patients undergoing general anesthesia. Raising the head bed by 10 to $20^{\circ}$ in patients after laparoscopic cholecystectomy with general anesthesia could significantly improve oxygenation after tracheal catheter removal. Nurses should instruct postoperative patients in a semi-recumbent position.

\section{References}

Chen, G. X. (2015). Effect of PEEP on respiratory function and atelectasis in obese patient after laparoscopic cholecystectomy. Nursing Practice and Research, 12(6), 3-4.

Coccolini, F., Catena, F., Pisano, M., Gheza, F., Fagiuoli, S., Di Saverio, S., \& Sartelli, M. (2015). Open versus laparoscopic cholecystectomy in acute cholecystitis. Systematic review and meta-analysis. International Journal of Surgery, 18, 196-204. doi:10.1016/j.ijsu.2015.04.083

Gao, M., Li, X. F., \& Cheng, Q. H. (2010). Effects of different postures on the measurement of central venous pressure in patients with mechanical ventilation. Chinese Journal of Nursing, 45(7), 622-623.

Jiang, W., Zhao, A., \& Liu, Y. (2013). Comparison on monitoring results of pulse and blood oxygen saturation between infusion limb and non-infusion limbs after general anesthesia operation. Nursing Research, 27(9),823-823.

Longo, M. A., Cavalheiro, B. T., \& de Oliveira Filho, G. R. (2017). Laparoscopic cholecystectomy under neuraxial anesthesia compared with general anesthesia: Systematic review and meta-analyses. Journal of Clinical Anesthesia, 41, 48-54. doi:10.1016/j.jclinane.2017.06.005

Nasser, M. F., Hussein, Y. M., Moustafa, M., Elsaye, E., Saffar, F., Al-Ghamdi, S. A., \& Sattar, S. A. (2015). Our experience of laparoscopic cholecystectomy at King Abdullah Hospital, Bisha: A retrospective Study. Pakistan Journal of Surgery, 31(3), 158-160.

Nathaniel, J. S., \& Preeti, M. (2018). Laparoscopic cholecystectomy. Retrieved from https://www.uptodate.com/contents/ laparoscopic-cholecystectomy\#H33

Pinto, V., Eriyawa, A., Weerasinghe, S., Senasinghe, R., \& Rajendran, V. (2013). Position adopted for the post-operative patient and effect on tidal volume. Sri Lankan Journal of Anesthesiology, 21(2), 64-67. doi:10.4038/slja.v21i2.5428

Saraswat, V. (2015). Effects of anesthesia techniques and drugs on pulmonary function. Indian Journal of Anesthesia, 59(9), 557. doi:10.4103/0019-5049.165850

Wolfe, B. M., Gardiner, B., \& Frey, C. F. (2015). Laparoscopic cholecystectomy: A remarkable development. JAMA, 314(13), 1406-1406. doi:10.1001/jama.2014.12014

Yousef, G. T., \& Lasheen, A. E. (2013). General anesthesia versus segmental thoracic or conventional lumbar spinal anesthesia for patients undergoing laparoscopic cholecystectomy. Anesthesia: Essays and Researches, 6(2), 167. doi:10.4103/0259-1162.108302

Zhang, L. (2014). Effects of different mechanical ventilation modes on plasma CC16 and respiratory function after laparoscopic surgery. Master's thesis, Hebei Medical University, Shijiazhuang, China.

Disclosure. This study did not receive any funding. CXL contributed to the study design, patient recruitment, data collection, and the writing of the first draft of the manuscript. QYL contributed to the study design, data analysis, and critical revision of the manuscript. GFH was responsible for patient recruitment, data collection and analysis, and revision of the manuscript. All authors contributed to and have approved the final manuscript. 